\title{
Serum levels of $\beta$-hydroxybutyrate and pyruvate, metabolic changes and cognitive function in patients with schizophrenia during antipsychotic treatment: a preliminary study
}

This article was published in the following Dove Press journal: Neuropsychiatric Disease and Treatment

\author{
Yu-Chi Huang, ${ }^{1-3}$ Pao-Yen \\ Lin, ${ }^{1,4}$ Yu Lee,' Chi-Fa Hung,' \\ Su-Ting Hsu, ${ }^{5}$ Chih-Ching \\ Wu, ${ }^{6,7, *}$ Liang-Jen Wang ${ }^{8, *}$
}

'Department of Psychiatry, Kaohsiung Chang Gung Memorial Hospital and Chang Gung University College of Medicine, Kaohsiung, Taiwan; ${ }^{2}$ Department of Nursing, Meiho University, Pingtung, Taiwan; ${ }^{3}$ Chung Shan Medical University School of Medicine, Taichung, Taiwan; ${ }^{4}$ Institute for Translational Research in Biomedical Sciences, Kaohsiuns Chang Gung Memorial Hospital, Kaohsiung, Taiwan; ${ }^{5}$ Department of Community Psychiatry, Kaohsiung Municipal Kai-Syuan Psychiatric Hospital, Kaohsiung, Taiwan; ${ }^{6}$ Molecular Medicine Research Center, Chang Gung University, Tao-Yuan, Taiwan; ${ }^{7}$ Department of Medical Biotechnology and Laboratory Science, Chang Gung University, Tao-Yuan, Taiwan; ${ }^{8}$ Department of Child and Adolescent Psychiatry, Kaohsiung Chang Gung Memorial Hospital, Chang Guns University College of Medicine, Kaohsiung, Taiwan

*These authors contributed equally to this work

Correspondence: Chih-Ching Wu Molecular Medicine Research Center, Chang Gung University, No 259, I Wen-Hua Road,

Tao-Yuan, Taiwan

Tel +886 32118800 ext 5093

Fax +886 32118800 ext 5247

Email luckywu@mail.cgu.edu.tw

Liang-Jen Wang

Department of Child and Adolescent

Psychiatry, Kaohsiung Chang Gung Memorial Hospital, No I23, Ta-Pei Road, Kaohsiung City,

Taiwan

Tel +886773 I 7123 ext 8753

Fax +8867732 6817

Email wangliangjen@gmail.com
Background: $\beta$-hydroxybutyrate $(\beta-\mathrm{HB})$ and pyruvate have been associated with the brain energy utilization, which may play a role in the pathophysiology of schizophrenia. In this prospective study, we aim to investigate the trends of $\beta$-HB and pyruvate levels, metabolic changes, and cognitive function in schizophrenia patients receiving antipsychotic treatment.

Objective: We recruited 38 schizophrenia patients who had been treated with antipsychotics for 12 weeks, as well as 38 healthy age- and gender-matched subjects. Blood samples were taken from the patients at baseline and week 12 to determine the serum levels of $\beta-\mathrm{HB}$, pyruvate, and metabolic parameters, while blood samples of the healthy controls were taken at baseline. We evaluated the psychopathology using the Positive and Negative Syndrome Scale and cognitive function using the Brief Assessment of Cognition in Schizophrenia.

Results: During the 12-week follow-up period, the $\beta$-HB levels in patients with schizophrenia showed a decreasing trend, particularly in those undergoing treatment with aripiprazole or ziprasidone. The serum levels of $\beta$-HB in patients at baseline and week 12 were both higher than the levels in the healthy controls. Among the schizophrenia patients, changes in $\beta$-HB were positively correlated with changes in executive function. On the other hand, serum pyruvate levels remained steady during the 12-week follow-up period, and we found no significant correlation between pyruvate changes and changes in cognitive function or clinical symptoms.

Conclusion: Our findings indicate that $\beta$-HB may possess a potential indicator of energy utilization and have a protective role in executive function in patients with schizophrenia. Additional longitudinal studies with a larger sample size and longer follow-up periods are necessary to identify the relationship of metabolite regulation and cognitive function during schizophrenia patients' exposure to antipsychotics.

Keywords: ketone body, energy, metabolism, executive function, cognition, antipsychotic, schizophrenia

\section{Introduction}

Neurocognitive deficits are a core manifestation in schizophrenia. ${ }^{1}$ Cognitive domain impairment affects patients' daily activities and can serve as a significant predictor of functional and treatment outcomes. ${ }^{2,3}$ Studies have suggested that comorbid metabolic disturbances may be contributing factors of cognition deficits, ${ }^{4}$ and impairments in cognitive function may have a reciprocal interaction with metabolic disturbances in schizophrenia patients. ${ }^{5}$ Nevertheless, the psychopathology underlying the communication between metabolism perturbation and cognitive dysfunction is complex and remains inconclusive. ${ }^{6}$ 
In normal conditions with sufficient oxygen, glucose is the primary energy substrate of the brain. Pyruvate is metabolized from glucose and then enters the tricarboxylic acid (TCA) cycle to create adenosine triphosphate (ATP) through the glycolysis process. ${ }^{7}$ In certain situations, such as when fasting or feeling stressed, ketone bodies become the brain's alternative energy substrate. ${ }^{8}$ Ketone bodies are derived from fatty acids through adipocyte lipolysis. ${ }^{9} \beta$-hydroxybutyrate $(\beta-\mathrm{HB})$ and acetoacetate, both of which are categorized as ketone bodies, are converted into acetoacetyl-CoA and then into acetyl-CoA, which enters the TCA cycle to generate ATP using the same final pathway as glucose. ${ }^{10}$ Ketone bodies are used by both neurons and glial cells in brain tissue. ${ }^{11}$ In particular, the levels of ketone bodies in the circulation system serve as a primary predictive factor of the energy utilization rate, ${ }^{9}$ and its correlation with the entire body's energy metabolism indicates that ketone body levels reflect dynamic energy metabolism changes.

Abnormalities in the brain's energy metabolism with regard to the regulatory pathways of fatty acid and glucose have been related to the pathophysiology of schizophrenia. ${ }^{12}$ In previous studies, researchers have reported a significantly altered membrane metabolism in the brain among first-episode, drug-naive schizophrenia patients who had a reduced precursor of membrane phospholipid phosphomonoesters and an increased breakdown of membrane phospholipid phosphodiesters compared with control subjects, which indicated hypoactivity of the dorsal prefrontal cortex (PFC) function when a metabolism disturbance occurs. ${ }^{13,14}$ Such a finding may indicate that the brain's energy fuel in schizophrenia patients tends to shift from glucose to ketone bodies under certain circumstances of brain glucose hypometabolism. ${ }^{15,16}$ If that is true, the demand for fatty acid is associated with the incremental breakdown of membrane phospholipids. Although pyruvate and $\beta-\mathrm{HB}$, which are 2 important energy substrates in the brain and circulation system, have been reported to be associated with the pathophysiology of schizophrenia, ${ }^{17} \beta$-HB has been further proposed as a potential indicator of energy utilization impairment in order to differentiate schizophrenia patients from healthy controls. ${ }^{18}$ Administering $\beta$-HB as energy substrates has been suggested as a method for correcting an energy metabolism deficiency of the brain. ${ }^{19}$ However, evidence evaluating the association between energy substrates and the brain's cognitive performance, as well as the correlation of their dynamic changes in patients undergoing schizophrenia and antipsychotic treatment is lacking.

Therefore, we have developed the following hypotheses. First, peripheral $\beta$-HB levels are higher in schizophrenia patients than in healthy controls to compensate for the energy metabolism deficiency in the acute phase of schizophrenia. Reduced $\beta$-HB levels are measured after the previous supplemental energy is effective, but $\beta$-HB levels still remain higher and differential from those of healthy control subjects. Second, cognitive function changes are correlated with the change of $\beta$-HB, rather than pyruvate, because $\beta$-HB possesses a potential indicator of energy utilization in schizophrenia patients. The purpose of this study was to examine $\beta$-HB and pyruvate levels at week 12 during treatment with antipsychotic drugs in comparison with healthy age- and gender-matched controls, as well as to investigate whether $\beta$-HB and pyruvate have varying trends in schizophrenia patients treated with different categories of antipsychotic drugs. Furthermore, we examined the differences in the changes of metabolic parameters and cognitive function related to various antipsychotic groups, as well as the potential correlation between $\beta$-HB and pyruvate and cognitive function.

\section{Methods \\ Participants}

This study received approval from the institutional review board (IRB) of Chang Gung Memorial Hospital (IRB No: 102-3977A3), and we obtained written informed consent from all the participants. The procedure of recruiting and assessing patients and healthy controls has already been described in detail elsewhere. ${ }^{18}$ In short, eligible patients with schizophrenia in an outpatient department of Chang Gung Memorial Hospital in Kaohsiung were eligible for this study if they met the following criteria: 1) aged 18-65 years; 2) diagnosed with schizophrenia as defined by the Diagnostic and Statistical Manual of Mental Disorders-Fourth Edition (Text Revision); ${ }^{20} 3$ ) being treated with an antipsychotic drug at a stable dose for at least 1 month before the study started with relatively stable psychotic symptoms; 4) with no history of major physical illnesses (such as genetic conditions, infectious conditions, cardiovascular diseases, or cancer), and 5) of Han Chinese ethnicity.

As for the control group, it consisted of healthy individuals recruited from staff of Kaohsiung Chang Gung Memorial Hospital and community volunteers in Kaohsiung City. The recruitment criteria for the control group included: 1) aged 18-65 years; 2) with no history of major psychiatric disorders (eg, psychosis, bipolar disorder, major depressive disorder, or organic mental disorders) or illicit drug use; 3) with no history of major physical illnesses (such as genetic, cardiovascular diseases, infectious conditions, or cancer); 4) of ethnic Han Chinese origin, and 5) age- and gender-matched with the 38 schizophrenia patients. 


\section{Antipsychotic treatment}

In this prospective open-label 12-week study, we examined the various effects of antipsychotics on $\beta$-HB, pyruvate, and cognitive function. We focused on comparing $\beta$-HB and pyruvate levels at week 12 with healthy control subjects and their possible association with cognitive function for patients being treated with different antipsychotics. We chose 12 weeks as a time point for follow-up based on previous research of detectable cognitive function changes in schizophrenia patients during the 12 -week period. . $^{21,22}$ Prior to starting the study, the patients were required to be treated with a stable dose of an antipsychotic drug for at least 1 month. The psychotic status of patients at the onset of the study also had to be relatively stable. During the 12-week follow-up period, the patients were maintained on their current dose of antipsychotics and were instructed not to change their lifestyles. We initially recruited 54 schizophrenia patients, and 38 of them completed the 12-week follow-up period. ${ }^{18}$ The antipsychotic drugs prescribed to these 38 patients were as follows: risperidone $(n=7)$; olanzapine $(n=6)$; quetiapine $(\mathrm{n}=1)$; amisulpride $(\mathrm{n}=5)$; sulpiride $(\mathrm{n}=1)$; aripiprazole $(\mathrm{n}=6)$; ziprasidone $(\mathrm{n}=1)$; paliperidone $(\mathrm{n}=2)$; and clozapine $(\mathrm{n}=9)$. Due to the small sample size, the patients were categorized into 3 groups based on the similar metabolic risks of the prescribed antipsychotics. ${ }^{23}$ The first group consisted of patients prescribed aripiprazole or ziprasidone (AZ group); the second group consisted of patients prescribed olanzapine or clozapine (OC group); and the third group consisted of patients prescribed any other antipsychotics (OA group). The dose of the antipsychotic drugs was re-calculated based on the defined daily dose recommended by the WHO Collaborating Centre for Drug Statistics Methodology. ${ }^{24}$ Afterward, 38 healthy subjects from the previous control groups were selected and age- and gender-matched with the schizophrenia patients. ${ }^{18}$

\section{Biochemical measurements}

We collected blood samples from the schizophrenia patients in the morning after they had fasted at baseline and week 12 . Serum levels of metabolite substrates, $\beta$-HB, and pyruvate were measured using the Colorimetric/Fluorometric Assay Kit from BioVision, Inc., Milpitas, CA, USA ( $\beta$-HB: sensitivity $0.01-0.2 \mathrm{mM}$; pyruvate: sensitivity $1 \mu \mathrm{M}$ to $10 \mathrm{mM}$, coefficient of variation $[\mathrm{CV}] 3.8 \%$ ). The metabolic parameters, namely the plasma levels of fasting glucose, triglycerides, total cholesterol, high-density lipoprotein-cholesterol (HDL-C), and low-density lipoprotein-cholesterol, were determined in the Laboratory Medicine Department of Chang Gung Memorial Hospital. We used $\beta$-HB and pyruvate levels from 38 healthy age- and gender-matched subjects for comparison.

\section{Clinical assessments}

We performed psychiatric diagnoses of both the schizophrenia patients and the healthy control subjects using the Mini International Neuropsychiatric Interview (MINI), a short, structured diagnostic interview, in order to assess psychiatric disorders. ${ }^{25}$ The MINI in Mandarin Chinese has been proven to have good reliability and has been widely used in international clinical trials and epidemiological studies. ${ }^{26}$ The psychopathology of schizophrenia patients was evaluated using the Positive and Negative Syndrome Scale (PANSS) score, which consists of 3 subscales that represent positive, negative, and general psychiatric symptoms. ${ }^{27} \mathrm{We}$ determined patients' age at onset and duration of illness through interviews with the patients and review of their medical records.

\section{Cognitive assessment}

We evaluated patients' cognitive functions using the Brief Assessment of Cognition in Schizophrenia (BACS), ${ }^{28}$ which consists of a battery of tests that measure the aspects of cognition that are most impaired and has been strongly correlated with the real-world functioning of schizophrenia patients. ${ }^{29}$ The norms and validity of the Chinese version of the BACS have already been demonstrated in previous studies. $^{30,31}$ The BACS tests consist of the List Learning Test, Digit Sequencing Task, Token Motor Task, Category Instances Test, Controlled Oral Word Association Test, Symbol Coding, and Tower of London Test, which assess verbal memory, working memory, motor speed, verbal fluency, attention, processing speed, and executive function, respectively. Afterwards, we calculated a composite score by comparing each patient's performance in each task with the performance of a healthy control subject, which was the $Z$-score or $T$-score of that sum. ${ }^{32} \mathrm{We}$ then used the $Z$-scores for each scale to perform our analyses.

\section{Statistical analysis}

We adopted the Statistical Package for the Social Sciences for Windows (version 21.0; SPSS, Inc., Chicago, IL, USA) to analyze the data. Variables are presented as either mean \pm SD or frequency.

We applied the Chi-square test or Fisher's exact test to compare categorical variables between schizophrenia patients and healthy controls, as well as among the 3 patient groups (AZ, OC, and OA groups). To compare the patient groups at baseline, descriptive statistics were performed using the Kruskal-Wallis test for metric variables. The independent $t$-test was used to compare the $\beta$-HB and pyruvate levels in the schizophrenia patients with those of the healthy controls. 
To explore the trends of metabolite substrates $(\beta-\mathrm{HB}$ and pyruvate) and clinical characteristics (BACS and PANSS scores) during the 12-week period, we repeatedly performed measurements of analysis of variance to examine the effects of time (12-week antipsychotic treatment) and group (AZ, OC, and OA groups), as well as any potential interactions. We hypothesized that antipsychotic treatment resulted in differential changes over 12 weeks in the 3 groups, which is supported by significant treatment $\times$ group interaction on dependent measures. We utilized Pearson's correlation to investigate the potential correlation between changes of metabolic parameters and metabolic substrates, as well as between metabolite substrate changes, cognitive function changes, and clinical symptoms during the 12 weeks. All the statistical tests were 2-tailed, and differences of $p<0.05$ were considered statistically significant.

\section{Results}

A total of 38 patients with schizophrenia (20 males and 18 females, mean age: $40.5 \pm 9.3$ years) completed the 12-week follow-up period, and 38 healthy controls were ageand gender-matched with schizophrenia patients (20 males and 18 females, mean age: $40.8 \pm 11.0$ years). Compared with the healthy controls, patients with schizophrenia were more likely to be unmarried and less educated, and have greater levels of body weight, body mass index, and triglycerides and a lower level of HDL-C. The age, sex, height, and other metabolic parameters did not differ significantly between these 2 groups (Table 1).

$\beta$-HB levels between baseline and week 12 in the schizophrenia patients demonstrated a statistically significant difference ( $\beta$-HB at baseline and week 12: $492.3 \pm 246.5 \mu \mathrm{M}$ vs $431.5 \pm 148.2 \mu \mathrm{M}, p=0.015)$. $\beta$-HB levels in the schizophrenia patients at baseline were found to be significantly higher than $\beta$-HB levels in the healthy controls $(492.3 \pm 246.5 \mu \mathrm{M}$ vs $327.4 \pm 100.0 \mu \mathrm{M}, p<0.001)$. At week 12 , the levels of $\beta$-HB in the schizophrenia patients remained significantly higher than the $\beta$-HB levels in the healthy controls $(431.5 \pm 148.2 \mu \mathrm{M}$ vs $327.4 \pm 100.0 \mu \mathrm{M}, p=0.001$; Figure $1 \mathrm{~A}$ ). The levels of pyruvate between baseline and week 12 in the schizophrenia patients demonstrated no statistically significant differ-

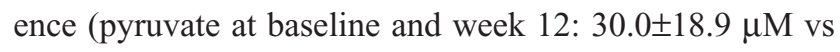
$29.3 \pm 13.9 \mu \mathrm{M}, p>0.05)$; moreover, the pyruvate levels in schizophrenia patients at baseline and week 12 showed no significant differences when compared with the pyruvate levels in the healthy controls $(25.7 \pm 9.0 \mu \mathrm{M}, p>0.05$; Figure 1B).

Of the 38 participating schizophrenia patients, 7 were treated with aripiprazole or ziprasidone (AZ group), 15 with olanzapine or clozapine (OC group), and 16 with other antipsychotics (OA group). We observed no significant differences

Table I Comparisons of demographic data, metabolic profiles and disease characteristics between patients with schizophrenia and healthy controls

\begin{tabular}{|c|c|c|c|c|}
\hline Variables & $\begin{array}{l}\text { Schizophrenia } \\
(n=38)\end{array}$ & $\begin{array}{l}\text { Healthy controls } \\
(n=38)\end{array}$ & $\begin{array}{l}\text { Statistic } \\
\text { value }\end{array}$ & $p$-value \\
\hline \multicolumn{5}{|l|}{ Demographic data } \\
\hline Sex (male/female), n (\%) & $20(52.6) / 18(47.4)$ & $20(52.6) / 18$ (47.4) & $\chi^{2}=0.000$ & 1.000 \\
\hline Age, years & $40.6 \pm 9.3$ & $40.8 \pm 11.0$ & $t=-0.135$ & 0.893 \\
\hline Education, years & $13.0 \pm 1.7$ & $15.1 \pm 3.2$ & $t=-3.646$ & $<0.00$ I $^{* * *}$ \\
\hline Married, n (\%) & $2(5.3)$ & $20(52.6)$ & $\chi^{2}=20.727$ & $<0.00 \mathrm{I}^{\mathrm{a}, * * * *}$ \\
\hline Height, cm & $165.6 \pm 8.8$ & $163.3 \pm 10.4$ & $t=1.019$ & 0.311 \\
\hline Body weight, kg & $74.4 \pm 14.6$ & $64.4 \pm 13.5$ & $t=3.098$ & $0.003 * *$ \\
\hline \multicolumn{5}{|l|}{ Metabolic profiles } \\
\hline Body mass index, $\mathrm{kg} / \mathrm{m}^{2}$ & $27.2 \pm 5.6$ & $24.0 \pm 3.2$ & $t=3.138$ & $0.002 * *$ \\
\hline Fasting glucose, mg/dL & $100.3 \pm 46.0$ & $86.1 \pm I I . I$ & $t=\mathrm{I} .860$ & 0.067 \\
\hline Total cholesterol, mg/dL & $173.7 \pm 40.7$ & $187.6 \pm 30.4$ & $t=-1.685$ & 0.096 \\
\hline Triglycerides, mg/dL & $127.3 \pm 65.5$ & $91.6 \pm 45.6$ & $t=2.762$ & $0.007 * *$ \\
\hline HDL-C, mg/dL & $48.2 \pm 12.7$ & $54.7 \pm 14.1$ & $t=-2.106$ & $0.039 *$ \\
\hline LDL-C, mg/dL & $103.7 \pm 29.7$ & $1 \mid 4.9 \pm 29.6$ & $t=-1.647$ & 0.104 \\
\hline \multicolumn{5}{|l|}{ Disease characteristics } \\
\hline Age of onset, years & $23.5 \pm 1.2$ & $\mathrm{~N} / \mathrm{A}$ & $\mathrm{N} / \mathrm{A}$ & $\mathrm{N} / \mathrm{A}$ \\
\hline Duration of illness, years & $16.9 \pm 1.6$ & N/A & $\mathrm{N} / \mathrm{A}$ & N/A \\
\hline DDD of antipsychotics & $1.2 \pm 0.2$ & $\mathrm{~N} / \mathrm{A}$ & $\mathrm{N} / \mathrm{A}$ & $\mathrm{N} / \mathrm{A}$ \\
\hline Duration of antipsychotic use, months & $41.4 \pm 7.8$ & N/A & N/A & $N / A$ \\
\hline
\end{tabular}

Notes: Data are expressed as mean \pm SD or $n(\%)$; ${ }^{p}$-value was calculated by using Fisher's exact test; ${ }^{*} p<0.05,{ }^{* *} p<0.01$, ${ }^{* * *} p<0.00 \mathrm{I}$.

Abbreviations: DDD, defined daily dose; HDL-C, high-density lipoprotein-cholesterol; LDL-C, low-density lipoprotein-cholesterol; N/A, not applicable. 


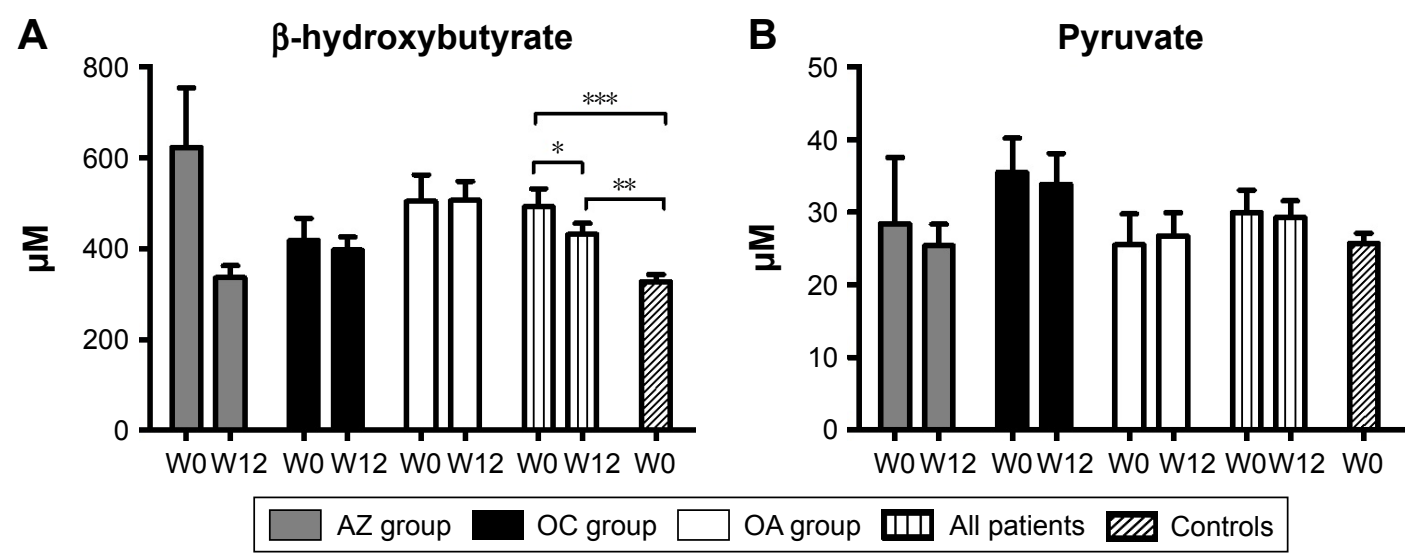

Figure I Changes of serum levels of $\beta-\mathrm{HB}(\mathbf{A})$ and pyruvate $(\mathbf{B})$ in patients with schizophrenia during a I2-week antipsychotic treatment period, and comparison with healthy controls.

Notes: $* p<0.05, * * p<0.01, * * * p<0.001 . \beta-H B$ levels in schizophrenia patients at baseline were significantly higher than $\beta$ - HB levels in healthy controls $(492.3 \pm 246.5$ vs $327.4 \pm 100.0 \mu \mathrm{M}, p<0.00 \mathrm{I})$. $\beta$-HB levels in patients at week 12 remained significantly higher than $\beta$-HB levels in the healthy controls $(43 \mathrm{I} .5 \pm I 48.2$ vs $327.4 \pm I 00.0 \mu \mathrm{M}$, $p=0.00$ I;A). Pyruvate levels between baseline and week I 2 in patients had no statistically significant difference ( $p y r u v a t e$ at baseline and week I 2:30.0 \pm I $8.9 \mathrm{vs} 29.3 \pm I 3.9 \mu \mathrm{M}, p>0.05$ ); pyruvate levels in patients at baseline and week 12 had no significant differences when compared with pyruvate levels in the healthy controls $(25.7 \pm 9.0 \mu \mathrm{M}, p>0.05$; $\mathbf{B})$.

Abbreviations: AZ group, patients being treated with aripiprazole or ziprasidone; $\beta$-HB, $\beta$-hydroxybutyrate; OA group, patients being treated with any other antipsychotics; OC group, patients being treated with olanzapine or clozapine.

with regard to demographic characteristics or metabolic parameters between the 3 patient groups at baseline $(p>0.05$; Table 2).

Table 3 lists the changes in metabolite substrates ( $\beta$-HB and pyruvate) and clinical characteristics (BACS and PANSS scores) over 12 weeks. Over the 12-week period, time $(\mathrm{F}=6.534, p=0.015)$ and treatment $\times$ group interaction $(\mathrm{F}=4.230, p=0.023)$ had a significant effect on $\beta$-HB serum levels. We observed an overall decreasing trend of serum $\beta$-HB levels in schizophrenia patients during the 12-week period of antipsychotic treatment. The decreasing trend of
$\beta$-HB levels was mainly the result of the AZ group. We found no significant time or group effect or treatment $\times$ group interaction on pyruvate.

As for cognitive function (Table 3), a significant group effect on verbal fluency $(\mathrm{F}=3.999, p=0.027)$ and a treatment $\times$ group interaction $(\mathrm{F}=4.141, p=0.024)$ was observed on executive function. The OC group had better verbal fluency performance than the $\mathrm{AZ}$ or OA groups. We further discovered that executive function measured by the BACS deteriorated in the $\mathrm{AZ}$ group over the 12 weeks, but not in the $\mathrm{OC}$ or OA groups.

Table 2 Comparisons of demographic data, metabolic profile and psychopathology between schizophrenic patients treated with different antipsychotics

\begin{tabular}{|c|c|c|c|c|c|}
\hline Variables & $\begin{array}{l}\text { Aripiprazole or } \\
\text { ziprasidone }(n=7)\end{array}$ & $\begin{array}{l}\text { Olanzapine or } \\
\text { clozapine }(n=15)\end{array}$ & $\begin{array}{l}\text { Other antipsychotics } \\
(n=16)\end{array}$ & $\begin{array}{l}\text { Statistic } \\
\text { value }\end{array}$ & $p$-value \\
\hline Sex (male/female), n (\%) & $2(28.6) / 5(7 \mid .4)$ & $9(60) / 6(40)$ & $9(56.3) / 7(43.8)$ & 2.036 & 0.440 \\
\hline Age, years & $37.1 \pm 10.5$ & $43.2 \pm 8.2$ & $39.3 \pm 9.7$ & $2.74 I$ & 0.254 \\
\hline Education, years & $13.4 \pm 2.5$ & $13.4 \pm 1.8$ & $12.3 \pm 1.1$ & 5.438 & 0.066 \\
\hline BMI, $\mathrm{kg} / \mathrm{m}^{2}$ & $26.5 \pm 6.4$ & $27.4 \pm 4.7$ & $27.4 \pm 6.4$ & 0.393 & 0.822 \\
\hline Fasting glucose, mg/dL & $117.9 \pm 105.6$ & $100.9 \pm 19.1$ & $92.1 \pm 14.0$ & 1.230 & 0.165 \\
\hline Total cholesterol, mg/dL & $164.4 \pm 15.5$ & $166.6 \pm 36.0$ & $184.4 \pm 50.8$ & 1.246 & 0.536 \\
\hline Triglycerides, mg/dL & $96.9 \pm 47.8$ & $144.9 \pm 80.7$ & $124.2 \pm 53.5$ & 2.777 & 0.249 \\
\hline HDL-C, mg/dL & $54.3 \pm 12.4$ & $43.7 \pm I I .0$ & $49.7 \pm 13.4$ & 3.760 & 0.153 \\
\hline LDL-C, mg/dL & $96.3 \pm 12.3$ & $97.4 \pm 27.6$ & $112.9 \pm 35.4$ & 1.931 & 0.381 \\
\hline Age of onset, years & $22.8 \pm 6.1$ & $22.7 \pm 7.8$ & $25.1 \pm 8.0$ & 0.868 & 0.648 \\
\hline Duration of illness, years & $14.5 \pm 9.2$ & $20.5 \pm 7.5$ & $14.6 \pm 10.9$ & 4.553 & 0.103 \\
\hline DDD of antipsychotics & $1.7 \pm 2.2$ & $1.1 \pm 0.5$ & $1.1 \pm 0.7$ & 0.510 & 0.775 \\
\hline Duration of antipsychotic use, months & $19.3 \pm 24.0$ & $57.5 \pm 56.1$ & $36.0 \pm 44.7$ & 2.919 & 0.232 \\
\hline
\end{tabular}

Notes: Data are expressed as mean \pm SD or $\mathrm{n}(\%)$; using Kruskal-Wallis test.

Abbreviations: BMI, body mass index; DDD, defined daily dose; HDL-C, high-density lipoprotein-cholesterol; LDL-C, low-density lipoprotein-cholesterol. 
Table 3 Changes of metabolite substrates, cognitive function and psychopathology in schizophrenia patients treated with different antipsychotics

\begin{tabular}{|c|c|c|c|c|c|c|c|c|c|}
\hline \multirow[t]{2}{*}{ Variables } & \multicolumn{2}{|c|}{$\begin{array}{l}\text { Aripiprazole or } \\
\text { ziprasidone }(n=7)\end{array}$} & \multicolumn{2}{|c|}{$\begin{array}{l}\text { Olanzapine or } \\
\text { clozapine }(n=15)\end{array}$} & \multicolumn{2}{|c|}{$\begin{array}{l}\text { Other antipsychotics } \\
(n=16)\end{array}$} & \multicolumn{3}{|c|}{ Statistic (f-values) } \\
\hline & Baseline & Week 12 & Baseline & Week 12 & Baseline & Week I2 & Time & Group & Time $\times$ group \\
\hline \multicolumn{10}{|l|}{ Metabolite substrates } \\
\hline$\beta$-hydroxybutyrate $(\mu \mathrm{M})$ & $623.7 \pm 346.3$ & $336.7 \pm 68.2$ & $417.9 \pm 190.2$ & $397.3 \pm 110.2$ & $504.6 \pm 233.1$ & $506.4 \pm|7| .5$ & $6.534^{*}$ & 1.531 & $4.230^{*}$ \\
\hline Pyruvate $(\mu \mathrm{M})$ & $28.4 \pm 25.4$ & $25.4 \pm 7.8$ & $35.5 \pm 18.3$ & $33.9 \pm 16.5$ & $25.5 \pm 17.0$ & $26.7 \pm 12.8$ & 0.123 & $\mathrm{I} .707$ & 0.159 \\
\hline \multicolumn{10}{|l|}{ Cognitive function (BACS) } \\
\hline Verbal memory & $-2.2 \pm 1.4$ & $-1.5 \pm 1.5$ & $-2.3 \pm 0.9$ & $-2.4 \pm I . I$ & $-2.2 \pm 1.8$ & $-2.4 \pm 1.1$ & 0.640 & 0.376 & 2.171 \\
\hline Working memory & $-1.4 \pm 1.2$ & $-1.4 \pm 0.7$ & $-1.2 \pm 1.0$ & $-1.0 \pm 1.2$ & $-1.8 \pm 1.3$ & $-1.5 \pm 1.6$ & 0.728 & 1.077 & 0.221 \\
\hline Motor speed & $-I . I \pm 0.9$ & $-0.5 \pm 1.0$ & $-1.4 \pm 1.2$ & $-1.5 \pm 1.0$ & $-I . I \pm 0.7$ & $-1.2 \pm 1.1$ & 1.205 & 1.215 & 2.128 \\
\hline Verbal fluency & $-2.8 \pm 0.6$ & $-2.6 \pm 0.7$ & $-2.2 \pm 0.7$ & $-2.3 \pm 0.4$ & $-2.8 \pm 0.4$ & $-2.6 \pm 0.7$ & 0.640 & $3.999 *$ & 0.911 \\
\hline Attention and processing speed & $-1.8 \pm 1.0$ & $-1.9 \pm 1.2$ & $-1.6 \pm 1.0$ & $-1.7 \pm 1.1$ & $-1.9 \pm 1.1$ & $-2.0 \pm 0.9$ & 1.809 & 0.447 & 0.038 \\
\hline Executive function & $-0.7 \pm 1.6$ & $-1.9 \pm 2.3$ & $-1.2 \pm 1.6$ & $-0.9 \pm 1.6$ & $-1.3 \pm 1.6$ & $-1.2 \pm 1.8$ & 1.664 & 0.118 & $\left.4.14\right|^{*}$ \\
\hline Composite score & $-2.7 \pm 1.5$ & $-2.7 \pm 1.4$ & $-2.6 \pm 1.0$ & $-2.6 \pm I . I$ & $-2.8 \pm 1.9$ & $-3.0 \pm 1.6$ & 0.005 & 0.218 & 0.134 \\
\hline \multicolumn{10}{|l|}{ Psychopathology } \\
\hline Positive symptoms score & $16.7 \pm 4.5$ & $17.0 \pm 6 . \mid$ & $18.9 \pm 5.9$ & $18.0 \pm 3.8$ & $17.0 \pm 4.0$ & $13.8 \pm 6.9$ & 1.657 & 1.856 & 1.111 \\
\hline Negative symptoms score & $17.4 \pm 5.6$ & $18.6 \pm 7.0$ & $22.5 \pm 8.8$ & $21.2 \pm 8.0$ & $19.4 \pm 6.1$ & $15.9 \pm 9.8$ & 1.163 & 1.435 & 1.280 \\
\hline General symptoms score & $35.6 \pm 8.5$ & $34.6 \pm 9.7$ & $42.6 \pm 10.2$ & $38.8 \pm 8.3$ & $39.4 \pm 9.1$ & $32.2 \pm 15.7$ & 0.053 & 1.396 & 0.463 \\
\hline Total score of PANSS & $69.7 \pm 16.4$ & $70.1 \pm 20.2$ & $84.1 \pm 22.0$ & $78.0 \pm 17.7$ & $75.8 \pm 15.8$ & $61.9 \pm 31.3$ & 3.016 & $1.76 \mid$ & 1.195 \\
\hline
\end{tabular}

Notes: Data are expressed as mean \pm SD or $n(\%) ; ~ * p<0.05$

Abbreviations: BACS, Brief Assessment of Cognition in Schizophrenia; PANSS, Positive and Negative Syndrome Scale.

In general, Pearson's correlation revealed that changes in $\beta$-HB in schizophrenia patients had a significant correlation with executive function changes over the 12-week period $(\mathrm{r}=0.424, p=0.008$; Figure 2). We observed no significant correlation between pyruvate and cognitive function changes or clinical symptoms during the 12-week period.

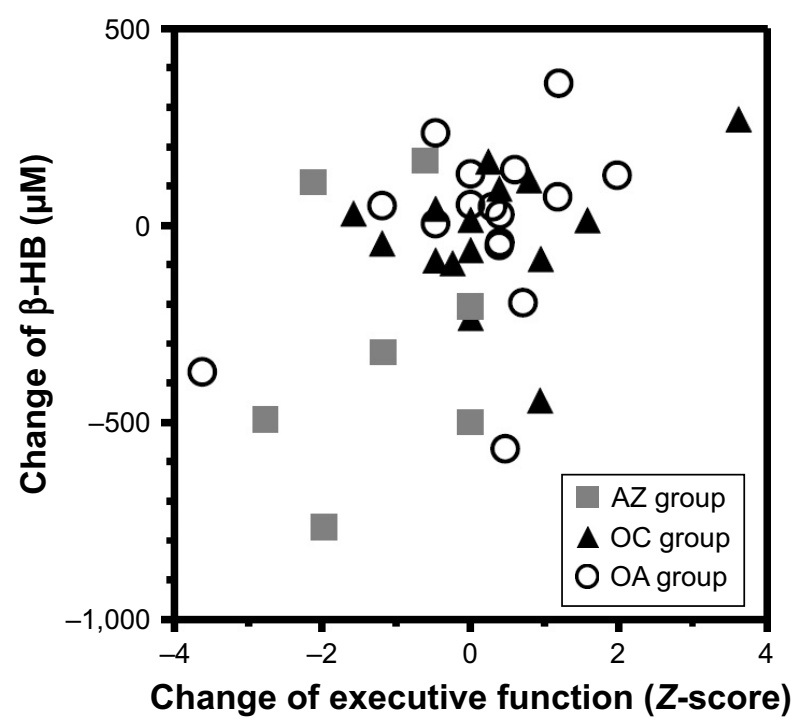

Figure 2 Scatterplot displaying the changes of $\beta-\mathrm{HB}$ and the changes of executive function during the 12-week antipsychotic treatment period $(r=0.424, p=0.008)$. Abbreviations: AZ group, patients being treated with aripiprazole or ziprasidone; $\beta$-HB, $\beta$-hydroxybutyrate; OA group, patients being treated with any other antipsychotics; OC group, patients being treated with olanzapine or clozapine.

\section{Discussion}

The main finding of this study is that we observed a significantly decreasing trend of serum $\beta$-HB levels in 38 schizophrenia patients during 12 weeks of antipsychotic treatment, but we also found that $\beta$-HB levels at week 12 remained significantly higher than those of healthy controls during the antipsychotic treatment. The previously mentioned decreasing trend of $\beta$-HB levels was primarily the result of aripiprazole or ziprasidone. Meanwhile, executive function tended to decrease over the 12 weeks of treatment in the AZ group. $\beta$-HB changes significantly correlated with executive function changes in all 38 medicated schizophrenia patients during the study period. However, serum pyruvate levels remained steady during the 12 -week antipsychotic treatment, and we found no significant correlation between pyruvate and cognitive function changes or clinical symptoms.

A previous study recruited neuroleptic-naïve schizophrenia patients to undergo 6-week monotherapy with risperidone, the results of which indicated a significantly declining trend during the 6 weeks. ${ }^{33}$ The general decreasing trend of $\beta$-HB levels during the 12 -week antipsychotic treatment in this study partially agrees with the aforementioned work, although medication status, antipsychotic treatment, and follow-up time varied. Furthermore, no significant time or group effect or treatment $\times$ group interaction was observed in relation to pyruvate. This result is in agreement with our 
hypothesis that decreasing $\beta$-HB levels could be observed following treatment with antipsychotics since the supplied energy partially improved the status of energy metabolism deficiency, but overall $\beta$-HB levels in schizophrenia patients remained differentially higher than those in healthy controls and, unlike pyruvate, can serve as a potential indicator of energy utilization.

Additional examination of the changes in $\beta$-HB levels in the 3 groups showed that the $\beta$-HB levels after the 12 -week treatment in the AZ group significantly decreased, and while $\beta$-HB changes in the OC group also presented a decreased trend, this finding did not reach statistical significance. Antipsychotic treatment has been associated with brain function and metabolism. ${ }^{34}$ Among them, a postsynaptic dopamine receptor antagonist could reduce ketonemia in a state of starvation. ${ }^{35}$ Aripiprazole is an atypical antipsychotic with partial agonist activity at dopamine $\mathrm{D}_{2}$ and serotonin $5 \mathrm{HT}_{1 \mathrm{~A}}$ receptors, as well as being antagonistic at $5-\mathrm{HT}_{2 \mathrm{~A}}$ receptors. ${ }^{36}$ Changes in $\beta$-HB are primarily observed in the AZ group; however, this finding cannot be explained by the previous finding that dopamine antagonist reduces ketonemia, which may hint that neurotransmitter dopamine may be involved in the energy utilization process but cannot explain the entire mechanism. Instead, the phenomenon of decreasing $\beta-\mathrm{HB}$ levels is more prominent in patients taking aripiprazole or ziprasidone, which are considered more "neutral" antipsychotics metabolically. The statistically indifferent dynamic change of $\beta$-HB levels in the OC group somewhat agrees with the result of another animal study which proposed that the plasma $\beta$-HB level is not affected by the use of olanzapine. ${ }^{37}$ In particular, the trend of reducing $\beta$-HB levels was significantly observed in patients prescribed antipsychotics with the least metabolic disturbance. This phenomenon suggests that the regulation of energy metabolism between fatty acids and glucose during antipsychotic treatment is complicated, and less metabolic perturbation caused by antipsychotics can reflect a more dominant energy utilization change via the energy substrate $\beta$-HB. The finding may also indicate the importance of peripheral $\beta$-HB levels in reflecting a variety of energy utilization between low vs high metabolism risks of antipsychotics due to their distinct effects. ${ }^{34}$ Due to the small sample size and short follow-up duration in this study, additional investigations will require a larger sample size and longer observation period in order to examine the effect of different antipsychotics on metabolite changes and the role of neurotransmitters in metabolism mechanisms.

As a treatment target of antipsychotics, executive function is vital to the functional outcome of schizophrenia. ${ }^{38}$
One previous study examined executive function in healthy controls by conducting a single dose of aripiprazole challenge and haloperidol challenge and comparing it with the placebo group. The results demonstrated a reducing trend in executive function performance in the aripiprazole group compared with the haloperidol and placebo groups. ${ }^{39}$ Another study demonstrated decreased brain metabolism in the frontal lobe of healthy subjects after a single dose of aripiprazole was administered, and the metabolic decline in the frontal lobe had a significant inverse correlation with striatal dopamine receptor occupancy and slower performance in cognitive tests. ${ }^{40}$ Furthermore, reduced executive function has also been observed after schizophrenia patients received aripiprazole adjunctive treatment concurrent to risperidone or olanzapine. ${ }^{41}$ Nevertheless, the efficacy of aripiprazole in treating cognitive dysfunction in schizophrenia patients is still inconclusive. A previous study has reported that aripiprazole has a better improvement effect on executive function than olanzapine ${ }^{42}$ or no difference compared with olanzapine. ${ }^{43}$ In the present study, executive function during the 12-week treatment period showed significant deterioration in the AZ group, but a similar trend was not observed in either the OC or OA groups. Meanwhile, patients prescribed olanzapine or clozapine demonstrated better verbal fluency. Other cognitive function subdomains, including motor speed, attention and processing speed, and composite score, remained stable during the 12 -week study period. Our findings indicate that different antipsychotics may vary with regard to their influence on certain cognitive function subdomains, rather than on global cognitive performance. However, a longer follow-up period is necessary to further evaluate the antipsychotic treatment response of cognitive impairment in schizophrenia patients.

The potential pathway of executive dysfunction in schizophrenia is connected to the PFC, as well as other brain regions, such as the hippocampus. ${ }^{44,45}$ One postmortem study found no difference in activity of the pyruvate dehydrogenase complex in dorsolateral PFC between schizophrenia patients and healthy controls and suggested that mitochondrial enzyme activity has less impact on brain metabolism in patients with schizophrenia. ${ }^{46}$ Our finding that peripheral pyruvate levels have no ability to differentiate schizophrenia patients from healthy controls and no significant correlation with executive function may agree in part with the aforementioned result. On the other hand, $\beta$-HB has been reported to protect cultured rat hippocampal neurons when exposed to a toxic environment with $\beta$ chain of amyloid precursor protein $\left(A \beta_{1-42}\right)$, a phenomenon related to memory 
impairment in Alzheimer's disease and that regulates the energy dysfunction of mitochondria. ${ }^{47}$ Higher $\beta$-HB levels are correlated with a greater degree of cognitive function improvement in memory-impaired adults being treated with medium-chain triglycerides for ketone body supplements. ${ }^{48}$ In our study, during the 12-week antipsychotic treatment period, changes in $\beta$-HB were significantly correlated with executive function changes. In other words, as $\beta$-HB level decreased, we observed that executive function deteriorated. This phenomenon may be partially explained by $\beta$-HB protecting cells against oxidative stress, which is associated with cognitive dysfunction. ${ }^{49}$ However, we have been unable to determine whether executive function is more susceptible to changes in energy metabolism than other cognitive function subdomains. This result suggests that $\beta$-HB may have a protective effect on executive function in schizophrenia patients undergoing antipsychotic treatment, while the significant correlation between $\beta$-HB and executive function may hint at $\beta$-HB serving as a marker for manifesting executive function. However, $\beta$-HB and pyruvate in the peripheral blood do not necessarily reflect their levels in the central nervous system. Further research is necessary to examine the impact of energy metabolites on cognitive function between different brain regions, especially PFC. Furthermore, a larger sample size and control for confounding factors, such as diet, exercise, and body weight changes would help further investigation into the relationship between $\beta$-HB levels and cognitive function.

This study has some limitations that should be mentioned. First, this work indicates a correlation between $\beta$-HB and executive function changes but cannot explain the possible causal relationship. We were unable to distinguish whether the changing $\beta$-HB levels were the result of exposure to antipsychotic treatment or the natural psychopathology of schizophrenia. Additional prospective studies could help to determine the influence of metabolites on cognitive function by controlling relevant confounding factors. Furthermore, we lacked the brain image data to further examine the connectivity between the PFC and other brain regions to differentiate executive function changes. ${ }^{45,50}$ Second, $\beta$-HB and pyruvate in peripheral blood are not perfect representations of their levels in the central nervous system. Additional research is required to examine the impact of energy metabolites in various brain regions on cognitive function. Third, the small sample size of each antipsychotic medication reduced the statistical power of our study. The positive correlation observed between changing levels of $\beta$-HB and changing cognitive function needs to be verified with individual antipsychotic medications by recruiting a larger sample size. Finally, our study lacked randomization and a placebo control group. The influence of pre-existing antipsychotic treatments on $\beta$-HB and pyruvate could not be ruled out due to the chronicity of the schizophrenia patients in this study.

\section{Conclusion}

While we observed a declining trend of serum $\beta$-HB levels in schizophrenia patients undergoing antipsychotic treatment, $\beta$-HB levels remained higher than those in healthy controls, and the change in $\beta$-HB levels was significantly correlated with executive function changes. This finding suggests that $\beta$-HB may manifest a protective role in the executive function of schizophrenia patients treated with antipsychotic medications. In the future, prospective studies with larger sample sizes and longer follow-up periods are necessary to further identify the relationship of metabolite regulation and cognitive function during schizophrenia patients' exposure to antipsychotics.

\section{Acknowledgment}

This study was supported by the Chang Gung Memorial Hospital Research Project (CMRPG8C1051 and CMRPG8C1291).

\section{Author contributions}

All authors contributed toward data analysis, drafting and revising the paper and agree to be accountable for all aspects of the work. CCW and LJW contributed equally as corresponding authors of this study.

\section{Disclosure}

The authors report no conflicts of interest in this work.

\section{References}

1. Harvey PD, Bowie CR, Friedman JI. Cognition in schizophrenia. Curr Psychiatry Rep. 2001;3(5):423-428.

2. Green MF. What are the functional consequences of neurocognitive deficits in schizophrenia? Am J Psychiatry. 1996;153(3):321-330.

3. Hoe M, Nakagami E, Green MF, Brekke JS. The causal relationships between neurocognition, social cognition and functional outcome over time in schizophrenia: a latent difference score approach. Psychol Med. 2012;42(11):2287-2299.

4. Kodl CT, Seaquist ER. Cognitive dysfunction and diabetes mellitus. Endocr Rev. 2008;29(4):494-511.

5. Bora E, Akdede BB, Alptekin K. The relationship between cognitive impairment in schizophrenia and metabolic syndrome: a systematic review and meta-analysis. Psychol Med. 2017;47(6):1030-1040.

6. O'Tuathaigh CM, Waddington JL. Mutant mouse models: phenotypic relationships to domains of psychopathology and pathobiology in schizophrenia. Schizophr Bull. 2010;36(2):243-245. 
7. Belanger M, Allaman I, Magistretti PJ. Brain energy metabolism: focus on astrocyte-neuron metabolic cooperation. Cell Metab. 2011;14(6): 724-738.

8. Owen OE, Morgan AP, Kemp HG, et al. Brain metabolism during fasting. J Clin Invest. 1967;46(10):1589-1595.

9. Williamson DH, Bates MW, Page MA, Krebs HA. Activities of enzymes involved in acetoacetate utilization in adult mammalian tissues. Biochem J. 1971;121(1):41-47.

10. Elwood JC, Marco A, Van Bruggen JT. Lipid metabolism in the diabetic rat. IV. Metabolism of acetate, acetoacetate, butyrate, and mevalonate in vitro. J Biol Chem. 1960;235:573-577.

11. Bentourkia M, Tremblay S, Pifferi F, Rousseau J, Lecomte R, Cunnane S. PET study of $11 \mathrm{C}$-acetoacetate kinetics in rat brain during dietary treatments affecting ketosis. Am J Physiol Endocrinol Metab. 2009; 296(4):E796-E801.

12. Prabakaran S, Swatton JE, Ryan MM, et al. Mitochondrial dysfunction in schizophrenia: evidence for compromised brain metabolism and oxidative stress. Mol Psychiatry. 2004;9(7):684-697, 643.

13. Pettegrew JW, Keshavan MS, Panchalingam K, et al. Alterations in brain high-energy phosphate and membrane phospholipid metabolism in first-episode, drug-naive schizophrenics. A pilot study of the dorsal prefrontal cortex by in vivo phosphorus 31 nuclear magnetic resonance spectroscopy. Arch Gen Psychiatry. 1991;48(6):563-568.

14. Keshavan MS, Pettegrew JW, Panchalingam KS, Kaplan D, Bozik E. Phosphorus 31 magnetic resonance spectroscopy detects altered brain metabolism before onset of schizophrenia. Arch Gen Psychiatry. 1991; 48(12):1112-1113

15. Desco M, Gispert JD, Reig S, et al. Cerebral metabolic patterns in chronic and recent-onset schizophrenia. Psychiatry Res. 2003;122(2):125-135.

16. Tamminga CA, Thaker GK, Buchanan R, et al. Limbic system abnormalities identified in schizophrenia using positron emission tomography with fluorodeoxyglucose and neocortical alterations with deficit syndrome. Arch Gen Psychiatry. 1992;49(7):522-530.

17. Yang J, Chen T, Sun L, et al. Potential metabolite markers of schizophrenia. Mol Psychiatry. 2013;18(1):67-78.

18. Huang YC, Lin PY, Lee Y, et al. $\beta$-hydroxybutyrate, pyruvate and metabolic profiles in patients with schizophrenia: a case control study. Psychoneuroendocrinology. 2016;73:1-8.

19. Cunnane SC, Courchesne-Loyer A, Vandenberghe C, et al. Can ketones help rescue brain fuel supply in later life? Implications for cognitive health during aging and the treatment of Alzheimer's disease. Front Mol Neurosci. 2016;9:53.

20. American Psychiatric Association. Diagnostic and Statistical Manual of Mental Disorders (DSM-IV-TR). Washington, DC: American Psychiatric Association; 2000.

21. Wang CH, Li Y, Yang J, et al. A randomized controlled trial of olanzapine improving memory deficits in Han Chinese patients with firstepisode schizophrenia. Schizophr Res. 2013;144(1-3):129-135.

22. Zhu W, Zhang Z, Qi J, et al. Adjunctive treatment for cognitive impairment in patients with chronic schizophrenia: a double-blind, placebocontrolled study. Neuropsychiatr Dis Treat. 2014;10:1317-1323.

23. Newcomer JW. Second-generation (atypical) antipsychotics and metabolic effects: a comprehensive literature review. CNS Drugs. 2005; 19(Suppl 1):1-93.

24. WHO collaborating centre for drug statistics methodology. ATC/DDD Index 2016. Available from: http://www.whocc.no/atc_ddd_index/. Accessed January 15, 2016.

25. Sheehan DV, Lecrubier Y, Sheehan KH, et al. The Mini-International Neuropsychiatric Interview (M.I.N.I.): the development and validation of a structured diagnostic psychiatric interview for DSM-IV and ICD-10. J Clin Psychiatry. 1998;59(Suppl 20):22-33; quiz 34-57.

26. Chou FH, Chou P, Su TT, et al. Quality of life and related risk factors in a Taiwanese Village population 21 months after an earthquake. Aust N Z J Psychiatry. 2004;38(5):358-364.

27. Kay SR, Opler LA, Lindenmayer JP. Reliability and validity of the positive and negative syndrome scale for schizophrenics. Psychiatry Res. 1988;23(1):99-110.
28. Keefe RS, Goldberg TE, Harvey PD, Gold JM, Poe MP, Coughenour L. The Brief Assessment of Cognition in Schizophrenia: reliability, sensitivity, and comparison with a standard neurocognitive battery. Schizophr Res. 2004;68(2-3):283-297.

29. Keefe RS, Poe M, Walker TM, Kang JW, Harvey PD. The Schizophrenia Cognition Rating Scale: an interview-based assessment and its relationship to cognition, real-world functioning, and functional capacity. Am J Psychiatry. 2006;163(3):426-432.

30. Wang LJ, Huang YC, Hung CF, et al. The Chinese version of the brief assessment of cognition in schizophrenia: data of a large-scale Mandarinspeaking population. Arch Clin Neuropsychol. 2017;32(3):289-296.

31. Wang LJ, Lin PY, Lee Y, et al. Validation of the Chinese version of brief assessment of cognition in schizophrenia. Neuropsychiatr Dis Treat. 2016;12:2819-2826.

32. Keefe RS, Harvey PD, Goldberg TE, et al. Norms and standardization of the brief assessment of cognition in schizophrenia (BACS). Schizophr Res. 2008;102(1-3):108-115.

33. Cai HL, Li HD, Yan XZ, et al. Metabolomic analysis of biochemical changes in the plasma and urine of first-episode neuroleptic-naive schizophrenia patients after treatment with risperidone. J Proteome Res. 2012;11(8):4338-4350.

34. Nenadic I, Dietzek M, Langbein K, et al. Effects of olanzapine on 31P MRS metabolic markers in schizophrenia. Hum Psychopharmacol. 2013;28(1):91-93.

35. Blesa-Malpica G, Johnston DG, Burrin JM, Orskov H, Heath AB, Alberti KG. Dopaminergic control of ketogenesis in fasting. Clin Endocrinol (Oxf). 1981;14(5):479-484.

36. McGavin JK, Goa KL. Aripiprazole. CNS Drugs. 2002;16(11):779-786; discussion 787-788.

37. Albaugh VL, Vary TC, Ilkayeva O, et al. Atypical antipsychotics rapidly and inappropriately switch peripheral fuel utilization to lipids, impairing metabolic flexibility in rodents. Schizophr Bull. 2012;38(1): $153-166$.

38. O'Grada C, Dinan T. Executive function in schizophrenia: what impact do antipsychotics have? Hum Psychopharmacol. 2007;22(6):397-406.

39. Bolstad I, Andreassen OA, Groote IR, Haatveit B, Server A, Jensen J. No difference in frontal cortical activity during an executive functioning task after acute doses of aripiprazole and haloperidol. Front Hum Neurosci. 2015;9:296.

40. Kim E, Howes OD, Turkheimer FE, et al. The relationship between antipsychotic D2 occupancy and change in frontal metabolism and working memory: a dual [(11)C]raclopride and [(18) F]FDG imaging study with aripiprazole. Psychopharmacology. 2013;227(2):221-229.

41. Yasui-Furukori N, Kaneda A, Sugawara N, Tomita T, Kaneko S. Effect of adjunctive treatment with aripiprazole to atypical antipsychotics on cognitive function in schizophrenia patients. J Psychopharmacol. 2012; 26(6):806-812.

42. Suzuki H, Gen K, Inoue Y. An unblinded comparison of the clinical and cognitive effects of switching from first-generation antipsychotics to aripiprazole, perospirone or olanzapine in patients with chronic schizophrenia. Prog Neuropsychopharmacol Biol Psychiatry. 2011; 35(1):161-168.

43. Kern RS, Green MF, Cornblatt BA, et al. The neurocognitive effects of aripiprazole: an open-label comparison with olanzapine. Psychopharmacology. 2006;187(3):312-320.

44. Molina V, Lubeiro A, Soto O, et al. Alterations in prefrontal connectivity in schizophrenia assessed using diffusion magnetic resonance imaging. Prog Neuropsychopharmacol Biol Psychiatry. 2017;76: 107-115.

45. Barch DM, Ceaser A. Cognition in schizophrenia: core psychological and neural mechanisms. Trends Cogn Sci. 2012;16(1):27-34.

46. Bubber P, Tang J, Haroutunian V, et al. Mitochondrial enzymes in schizophrenia. J Mole Neurosci. 2004;24(2):315-321.

47. Kashiwaya Y, Takeshima T, Mori N, Nakashima K, Clarke K, Veech RL. D-beta-hydroxybutyrate protects neurons in models of Alzheimer's and Parkinson's disease. Proc Natl Acad Sci U S A. 2000;97(10): 5440-5444. 
48. Reger MA, Henderson ST, Hale C, et al. Effects of beta-hydroxybutyrate on cognition in memory-impaired adults. Neurobiol Aging. 2004;25(3):311-314.

49. Shimazu T, Hirschey MD, Newman J, et al. Suppression of oxidative stress by beta-hydroxybutyrate, an endogenous histone deacetylase inhibitor. Science. 2013;339(6116):211-214.
50. Giraldo-Chica M, Rogers BP, Damon SM, Landman BA, Woodward ND. Prefrontal-thalamic anatomical connectivity and executive cognitive function in schizophrenia. Biol Psychiatry. Epub 2017 Sep 28.

\section{Publish your work in this journal}

Neuropsychiatric Disease and Treatment is an international, peerreviewed journal of clinical therapeutics and pharmacology focusing on concise rapid reporting of clinical or pre-clinical studies on a range of neuropsychiatric and neurological disorders. This journal is indexed on PubMed Central, the 'PsycINFO' database and CAS, and is the official journal of The International Neuropsychiatric Association (INA). The manuscript management system is completely online and includes a very quick and fair peer-review system, which is all easy to use. Visit http://www.dovepress.com/testimonials.php to read real quotes from published authors.

\footnotetext{
Submit your manuscript here: http://www.dovepress.com/neuropsychiatric-disease-and-treatment-journal
} 